OPEN ACCESS

Edited by:

Eleonore Fröhlich,

Medical University of Graz, Austria

Reviewed by:

Amitava Mukherjee,

VIT University, India

Martijn J. Schuijs,

University of Cambridge,

United Kingdom

*Correspondence:

Guimiao Lin

gmlin@szu.edu.cn

Specialty section: This article was submitted to

Predictive Toxicology,

a section of the journal

Frontiers in Pharmacology

Received: 16 January 2020 Accepted: 24 July 2020

Published: 17 August 2020

Citation:

Yang Z, Pan Y, Chen T, Li L, Zou W, Liu D, Xue D, Wang X and Lin G (2020)

Cytotoxicity and Immune Dysfunction

of Dendritic Cells Caused

by Graphene Oxide.

Front. Pharmacol. 11:1206.

doi: 10.3389/fphar.2020.01206

\section{Cytotoxicity and Immune Dysfunction of Dendritic Cells Caused by Graphene Oxide}

\author{
Zhiwen Yang ${ }^{1}$, Yongning Pan ${ }^{2}$, Tingting Chen ${ }^{1}$, Li Li ${ }^{1}$, Wenyi Zou ${ }^{1}$, Dongmeng Liu ${ }^{1}$, \\ Dahui Xue ${ }^{1}$, Xiaomei Wang ${ }^{1}$ and Guimiao Lin $^{1 *}$

\begin{abstract}
1 Base for International Science and Technology Cooperation: Carson Cancer Stem Cell Vaccines R\&D Center, Shenzhen Key Lab of Synthetic Biology, Department of Physiology, School of Basic Medical Sciences, Shenzhen University, Shenzhen, China, ${ }^{2}$ Department of Disease Prevention and Control, Shenzhen Baoan District Health Bureau, Shenzhen, China
\end{abstract}

Graphene, known as "black gold", has important applications in various fields. In previous studies, it has been proved that graphene oxide (GO) which is a derivative of graphene has low toxicity. However, the immunotoxicity of GO has not been fully elucidated. In this work, we used DC2.4 cell line to investigate the in vitro immunotoxicity of two types of GO, mono-layer GO (mono-GO) and multi-layer GO (multi- GO). We found that mono-GO had less effect on cell viability than multi-GO, but both mono-GO and multi-GO significantly induced the generation of ROS in DC2.4 cells. Interestingly, mono-GO caused DC2.4 cells to aggregate, thus changed the cell morphology significantly. However, no similar influence occurred for multi-GO. In addition, the results showed that these two GOs obviously enhance the release of TNF- $\alpha$ by DC2.4 cells with and without LPS stimulation. GO did not affect the level of IL-6 released from DC2.4 cells, but multi-GO promoted the release of IL-6 while mono-GO inhibited the production of IL-6 when cells were in response to LPS stimulation. Whole-transcriptome sequencing analysis found some immune-related differentially expressed genes including H2-DMb1, Ncbp3, Oas2, Men1, Fas, Cd320, Cd244, and Tinagl1 which are engaged in the immune system process. These results suggested that both mono-GO and multi-GO are immunotoxic to DC2.4 cells, which provides important basis for subsequent biological and clinical medical applications.

Keywords: graphene oxide, immunotoxicity, reactive oxygen species, dendritic cells, cytokines

\section{INTRODUCTION}

Graphene, called the "21st century materials", is a 2D nanomaterial composed of a single layer of sp2 carbon atoms (Carrow et al., 2018). Graphene can be divided into different types according to its functional form (Novoselov et al., 2004). Among them, graphene oxide (GO) is an oxidized form of graphene containing various oxygen-containing functional groups, such as carboxyl, carbonyl and hydroxyl (Gao, 2015). Since GO has a large surface area, and good physical, chemical and biological properties (Reina et al., 2017), they are considered for biological applications such as bioimaging, diagnostics, biosensing, photothermal and photodynamic therapy, tissue engineering, and drug 
delivery (Nurunnabi et al., 2015). Han et al. demonstrated that GO can be used as natural antioxidants to reduce inflammation by reducing reactive oxygen species (ROS) in macrophages and used as a gene carrier to synergistically treat myocardial infarction (Han J. et al., 2018). In addition, Zhang et al. proposed a GO-based fluorescent nanosensor that can be used to quickly detect telomerase content in cells and image telomerase (Zhang et al., 2018). Zhou et al. studied a new method of introducing GO into bone tissue to enhance biomineralization, and they found that when the GO concentration was $0.1 \% \mathrm{w} / \mathrm{v}$ and immersed in simulated body fluids for 7 days, GO-collagen-apatite 3D scaffold showed good therapeutic effect in repairing skull defects in rats (Zhou et al., 2018).

Although GO has a good application prospect, its unknown toxicity is still a bottleneck to clinical application. In addition, with the widespread use of GO, there are a variety of indirect and direct pathways to introduce them into the environment (e.g., dust or wastewater) (Markovic et al., 2018). Therefore, studies on the toxicity of GO need to be urgently addressed. In the recent years, $\mathrm{Qu}$ et al. summarized the toxic effects of graphene-family nanomaterials (GFNs) in cell and organs models and pointed out that $\mathrm{GO}$ can be transmitted to bodies through oral administration, intraperitoneal injection and intravenous injection and thus cause various inflammatory reactions (Ou et al., 2016). The toxicological mechanisms of GFNs mainly contain inflammatory response, necrosis apoptosis, DNA damage, and autophagy etc. For example, grapheme oxide caused a significant increase in intracellular ROS production in human HaCaT skin keratinoyctes, which was mainly mediated by activation of flavin-based oxidase, and caused mitochondrial membrane depolarization and mitochondrial damage (Pelin et al., 2018). Although many in vitro and in vivo toxicity studies of graphene have been reported, little research has been done on the immune system.

The immune system plays an important role in identifying and eliminating foreign pathogens. Immune cells can directly contact nanomaterials and are responsible for potential adverse reactions to GO. The results of research on the interaction between nanomaterials and the immune system can better assess the impact of GO on the human body. Recently, publications on the effects of GO on the immune system have focused on the study of macrophages (Dudek et al., 2016). Luo et al. found that PEGylated GO nanosheets interacted with peritoneal macrophage surface receptors by adsorbing to and/ or partially inserted into the cell membrane, and stimulated peritoneal macrophages to produce cytokine responses (Luo et al., 2017). Hoyle et al. found that GO had no significant inflammatory response to macrophages cells. However, GO inhibited Toll-like receptor 4 (TLR4) receptor-mediated interleukin production, but did not inhibit activation of inflammatory bodies (Hoyle et al., 2018). However, little is known about the effect of GO on dendritic cells (DCs), which were the most powerful professional antigen-presenting cells (APCs). Li et al. found that GOx nanosheets could be used to adsorb proteins. When the ovalbumin antigen binds to GOx, it could be internalized by DCs and initiated the antigen presentation reaction. Therefore, GOx nanosheets could be used as nanocarriers in vaccine formulations (Li et al., 2016). DCs drive specific responses through the adaptive immune system and plays a major role in innate immunity. DCs can efficiently ingest, process, and present antigens. Immature DCs have a strong ability to migrate. Mature DCs highly express MHC molecules and co-stimulatory molecules, and activate the native $\mathrm{T}$ cells, which in turn causes an immune regulatory response (Qian and Cao, 2018). DCs recognizes microbial components such as lipopolysaccharide (LPS) and specifically binds TLR4 on DCs (Chen et al., 2020). After LPS binds to TLR4, DCs secrete inflammatory factors such as tumor necrosis factor$\alpha$ (TNF- $\alpha$ ) and interleukin-6 (IL-6) (Fu et al., 2011; Chen et al., 2020). Accordingly, here we studied the immunotoxicity of mono-GO and multi-GO on DCs.

In this work, we utilized dendritic cell (DCs) line DC2.4 cells as an in vitro model to study the immunotoxicity of mono-GO and multi-GO. We found that multi-GO caused stronger toxicity to DC2.4 cells than mono-GO. And they all promoted the production of ROS in DC2.4 cells. Interestingly, mono-GO will cause a significant change in cell morphology, compared to multi-GO. In addition, mono-GO and multi-GO alone did not cause DC2.4 cells to produce IL-6, but stimulated DC2.4 cells to produce TNF- $\alpha$. However, after pretreatment with mono-GO and then treating DC2.4 cells with LPS, it was found that the cytokines secreted by DC2.4 cells were disordered. Also, we utilized RNA-seq found that both mono-GO and multi-GO were able to arouse immune responses. Our results showed that GO were able to disturb the immune function of DCs, which provided a basis for further applications and research.

\section{MATERIALS AND METHODS}

\section{Preparation and Characterization of GO}

Mono-GO (777676) and multi-GO (796034) were purchased from Sigma-Aldrich Technology. The absorption spectra of mono-GO and multi-GO were determinated by UV-Vis spectrophotometer (Beckman Coulter, DU720) and the morphology images of mono-GO and multi-GO in water were obtained with a transmission electron microscope (TEM) (JEOL model jem-2100, Test Center of Wuhan University). Zetasizer Nano ZS device (Malvern, U.K.) was used to measure the particle size and potential.

\section{Cell Culture}

The mouse dendritic cell line (DC2.4) were obtained in our lab. The DC2.4 cell line was cultured in RPMI 1640 (Gibco, USA) supplemented with $10 \%$ fetal bovine serum (FBS, Gibco, USA) and $100 \mathrm{U}$ penicillin/streptomycin (PS, Gibco, USA). All cells were cultured at $37^{\circ} \mathrm{C}$ in humidified atmosphere with $5 \% \mathrm{CO}_{2}$. One $\mu \mathrm{g} / \mathrm{ml}$ of lipopolysaccharides (LPS, L4391, Sigma, USA) in PBS was used as a positive control to stimulate the DC2.4 cells to secrete inflammatory factors. 


\section{Cell Viability}

The cell viability of DC2.4 cells treated with mono-GO and multi-GO was determined by MTT (Thiazolyl Blue Tetrazolium Bromide, M5655, Sigma, USA) assay. Briefly, DC2.4 cells were seeded in two 96-well plates at a density of $1.5 \times 10^{4}$ cells per well. Twelve hours later, $0.01,0.1,1,10$, or $100 \mu \mathrm{g} / \mathrm{ml}$ mono-GO and multi-GO in water were added to 96-well plates. After $24 \mathrm{~h}$ or $48 \mathrm{~h}$ incubation with cells, $20 \mu \mathrm{l}$ of MTT at a concentration of $5 \mathrm{mg} / \mathrm{ml}$ in PBS was added to each well, and the DC2.4 cells were subsequently cultured for $4 \mathrm{~h}$ in a cell incubator. The supernatant was then gently aspirated and $150 \mu \mathrm{l}$ dimethylsulfoxide (DMSO, D2650, Sigma, USA)was added to dissolve the pellet. The 96-well plates were placed on a shaker and shaken for $5 \mathrm{~min}$ at room temperature. Finally, we used a microplate reader (BioTek, USA) to measure the absorbance of each well at a wavelength of 490 $\mathrm{nm}$. All experiments were repeated three times. The cell viability was calculated by the absorbance of the sample wells against the control wells, assigning the viability of non-treated cells as $100 \%$.

\section{Enzyme-Linked Immunosorbent (ELISA) Assay}

To analyze the cytokine secretion by DC2.4 cells, $1 \times 10^{5}$ DC2.4 cells were seeded in 24-well plates-and cultured for $24 \mathrm{~h}$. Two kinds of GOs were added in $1 \mathrm{ml}$ RPMI 1640 with 10\% FBS and added to cells in each well at different concentrations, respectively0, $0.01,0.1,1,10,100 \mu \mathrm{g} / \mathrm{ml}$, then the cells were incubated for $4 \mathrm{~h}$ and then $1 \mu \mathrm{g} / \mathrm{ml}$ Lipopolysaccharide (LPS) was added. So far, the experiment was divided into six groups: negative control group, LPS positive control group, mono-GO group, multi-GO group, mono-GO+LPS group and multi-GO + LPS group. After the DC2.4 cells were co-cultured for 24 and $48 \mathrm{~h}$, the supernatant from the above six groups was collected and the cytokines (TNF- $\alpha$, IL-6) were measured by mouse ELISA kits (88-7324-88 and 88-7064-88, Invitrogen, Thermo Fisher Scientific) according to the manufacturer's instructions.

\section{DC2.4 Cells Morphology}

First, $2.5 \times 10^{5}$ DC2.4 cells were seeded in a 12-well plate. After $12 \mathrm{~h}$, DC2.4 cells were treated with $10 \mu \mathrm{g} / \mathrm{ml}$ mono-GO for $24 \mathrm{~h}$, and then rinsed briefly in phosphate-buffered saline (PBS). Next, 4\% paraformaldehyde was used to fix the DC2.4 cells for about 10 min and ice-cold PBS was used to wash the cells. And then, DC2.4 cells were incubated with $0.1 \%$ Triton X-100 for about 10 min and incubated with phalloidin-FITC (P5282, sigma, USA) antibody in PBS for $40 \mathrm{~min}$ at room temperature in the dark, and then washed with PBS three times with $5 \mathrm{~min}$ for each wash. Finally, DC2.4 cells were treated with DAPI for $1 \mathrm{~min}$ and images were obtained with a fluorescence microscope (X-Cite Series 120, Zeiss, Germany).

\section{Measurement of Reactive Oxygen Species (ROS) in Dendritic Cells}

ROS production by DC2.4 cells exposed to mono-GO and multiGO was evaluated by the 2, 7-dichlorofluorescindiacetate (DCFDA) (D6883, Sigma, USA) assay. Briefly, $2 \times 10^{4}$ DC2.4 cells were seeded in 96 -well clear bottom black side plate and cultured at $37^{\circ} \mathrm{C}$. After $12 \mathrm{~h}, 20 \mu \mathrm{M}$ DCFDA probe in serum-free medium was added to different wells every $30 \mathrm{~min}$ as a control group. At the same time, DCFDA probe and $10 \mu \mathrm{g} / \mathrm{ml}$ mono-GO or $10 \mu \mathrm{g} / \mathrm{ml}$ multi-GO in serum-free medium were added to different wells every $30 \mathrm{~min}$ as two experimental groups. Next, a fluorescence microplate reader (Infinite 200 Pro, Tecan) was used to read fluorescence at an excitation wavelength of $488 \mathrm{~nm}$ and an emission wavelength of $525 \mathrm{~nm}$. The average fluorescence intensity of intracellular DCF reflected the production of intracellular ROS.

\section{Whole-Transcriptome Sequencing Analysis (RNA-Seq)}

DC2.4 cells were cultured in 6-well plate at a density of $3 \times 10^{5}$ cells per well in $2 \mathrm{ml}$ of RPMI 1640 with 10\% FBS. After $12 \mathrm{~h}$, DC2.4 cells were treated with $10 \mu \mathrm{g} / \mathrm{ml}$ mono-GO and multi-GO for $24 \mathrm{~h}$, respectively. The supernatant was removed and the cells were washed twice with ice-cold PBS, then the cells were lysed in 500 $\mu \mathrm{l}$ TRIzol reagent (MRC, USA) to extract RNA, according to the manufacturer's protocol. Then Agilent 2100 Bioanalyzer (Agilent Technologies, Palo Alto, CA, USA) was used to assess RNA integrity and quantify RNA concentration. The screening conditions for RNA samples are: $28 \mathrm{~S} / 18 \mathrm{~S}$ rRNA band intensity is $2: 1$, spectral A260/A280 nm ratio is $1.8-2.0$ and A260/A230 nm ratio is greater than 1.5. The next step is to construct a gene library. In short, magnetic beads with Oligo (dT) are used to enrich mRNA. Subsequently, the mRNA is broken into short fragments, and the mRNA is used as a template for reverse transcription to synthesize the first-strand cDNA and then the second-strand cDNA. Then use AMPure XP beads to purify the double-stranded CDNA, modify the ends to connect the sequencing adapter, and finally perform PCR amplification to obtain the final gene library. Finally, the Illumina high-throughput sequencing platform (HiSeq/MiSeq) was used for paired-end sequencing. Finally, samples were sent to Guangzhou MAGIGENE Gene Corporation for subsequent analysis. Genes which $\mathrm{p}<0.05$ and $\log _{2}$ fold change $<-1$ or $>1$ were considered to be statistically significant and were filtered out for subsequent analysis between mono-GO, multi-GO and untreated groups. Gene Ontology (GO) enrichment analysis contains three GO terms which are biological processes (BP), molecular functions (MF), and cellular components (CC). In our study, the Kyoto Encyclopedia of Genes and Genomes (KEGG) database was used to analyze which important pathways of DCs can be changed by two GOs.

\section{Statistical Analysis}

We used SPSS 13.0 software for statistical analysis. Statistical evaluations were performed using independent $t$-test and oneway analysis of variance (ANOVA) test when normality and homogeneity of variance are satisfied. The results are presented as mean $\pm \mathrm{SD}$ values. All tests were two-sided and $\mathrm{P}<0.05$ was considered statistically significant.

\section{RESULTS}

\section{Characterization of GO}

The mono-GO and multi-GO were characterized with UV-Vis and TEM. The absorption spectrum of mono-GO was shown in 
Figure 1A. The absorption spectrum of multi-GO was shown in Figure 1B. As is shown in Figures 1A, B, the multi-GO has a wider absorption band than the mono-GO. The TEM image of mono-GO and multi-GO was shown in Figures 1C, D. It demonstrated that the lateral size of mono-GO is larger than that of multi-GO. It is clearly seen that the multi-GO is relatively thick due to stacking together, and the mono-GO is thin and transparent. The lateral size of mono-GO was larger than multiGO. In order to more clearly study the differences between mono-GO and multi-GO, we used a Zetasizer Nano ZS device (Malvern, U.K.) to measure the particle size and potential. Research showed that the zeta potentials of mono-GO and multi-GO were $-28.5 \pm 0.70$ and $-33.4 \pm 0.41$, respectively. The zeta potential values of our graphene oxides are similar to those found by other authors (Li et al., 2014; Hidalgo et al., 2015) The results indicated that mono-GO and multi-GO were relatively stable in water. The particle sizes of mono-GO and multi-GO were $1554.00 \pm 543.34 \mathrm{~nm}$ and $123.48 \pm 47.95 \mathrm{~nm}$, respectively. The results show that the particle size of mono-GO is much larger than that of multi-GO.

\section{Cytotoxicity Assay}

The effect of mono-GO and multi-GO on DC2.4 cell viability was evaluated by MTT assay after 24 and $48 \mathrm{~h}$ exposure time. As shown in Figure 2A, treatment of DC2.4 cells with different concentrations of mono-GO $(0.01,0.1,1,10,100 \mu \mathrm{g} / \mathrm{ml})$ for $24 \mathrm{~h}$ had no significant effect on the viability of DC2.4 cells. However, the viability of DC2.4 cells with mono-GO treatment for $48 \mathrm{~h}$ decreased obviously when the concentration reached up to 10 $\mu \mathrm{g} / \mathrm{ml}$. Different from mono-GO DC2.4 cell viability after multiGO treatment decreased remarkably when the concentration was higher than $0.01 \mu \mathrm{g} / \mathrm{ml}$. With the increasing concentration of
A

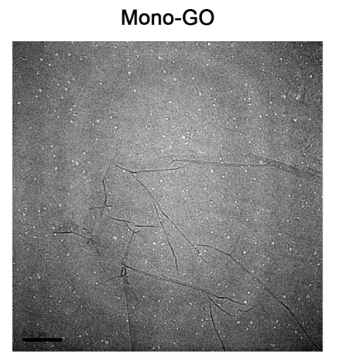

B

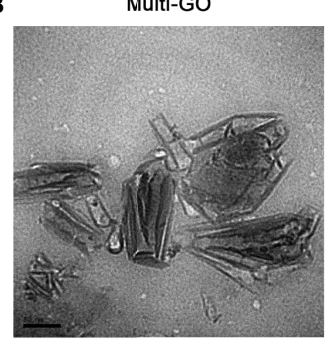

C

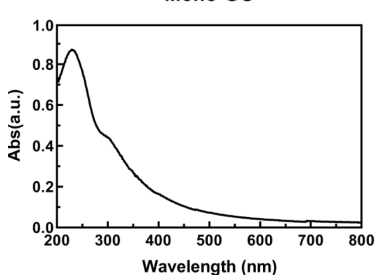

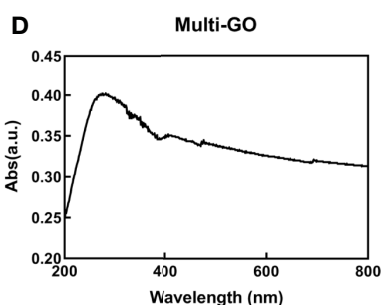

FIGURE 1 | Characterization of mono-graphene oxide (GO) and multi-GO. (A) Transmission electron microscope (TEM) image of multi-GO. Scale bar: 100 nm; (B) TEM image of mono-GO. Scale bar: 200 nm; (C) UV-Vis spectrum of multi-GO; (D) UV-Vis spectrum of mono-GO.
multi-GO, the cell viability decreased significantly on dosedependent manner. These results suggested that mono-GO had lower toxicity effect on DC2.4 cell viability than multi-GO.

The effect of GO on the morphology of DC2.4 cells was observed with a microscope. As shown in Figure 2B, after DC2.4 cells were exposed to $10 \mu \mathrm{g} / \mathrm{ml}$ mono-GO for $24 \mathrm{~h}$, the cells tend to aggregate and the cell morphology changed obviously. However, DC2.4 cells with multi-GO treatment showed no similar cell morphology alteration (Figure 2B). Therefore, we further observed the cytoskeleton of mono-GO treated cells by phalloidin-FITC immunofluorescence assay. We found that the cells became larger and the cytoplasm became fuller after monoGO treatment in comparison with those without treatment (Figure 2C).

To deeply investigate the effects of mono-GO and multi-GO on DCs, the kinetics of ROS production was investigated at increasing intervals of time (30 to 120 min exposure) using a time-dependent DCFDA assay. As is shown in Figure 2D, when compared with control, ROS production by DCs treated with mono-GO and multiGO significantly increased at the concentration of $10 \mu \mathrm{g} / \mathrm{ml}$. In addition, GO-induced ROS production was time-dependent from $30 \mathrm{~min}$ to $120 \mathrm{~min}$. Interestingly, mono-GO-induced ROS production was more than multi-GO after 30 and $120 \mathrm{~min}$ exposure. These results suggested that mono-GO and multi-GO had the potential to induce the intracellular ROS generation.

\section{Cytokine Secretion Assay of DC2.4 Cells Exposed to Mono-GO and Multi-GO}

To further observe the effect of mono-GO and multi-GO on the function of DCs, the production of the pro-inflammatory cytokine IL- 6 and TNF- $\alpha$ were tested in the culture supernatants of DC2.4 cells after 24 h-exposure to mono-GO and multi-GO. The bacterial endotoxin LPS which is known to induce the release of inflammatory factors in DC2.4 cells were used as positive control. As shown in Figure 3, LPS remarkably induced the release of the pro-inflammatory cytokines IL- 6 and TNF- $\alpha$ in DC2.4 cells. However, Mono-GO and multi-GO hardly induced the release of the IL-6 (Figures 3A-D), while obviously increased the production of TNF- $\alpha$ from DC2.4 cells with increasing concentration (Figures 3E, H).

To analyze the effect of GO on the immune response by DC2.4 cells, we further evaluated stress in DC2.4 cells caused by foreign materials. As the results of Figures 4A, B show, after pretreatment with mono-GO, the release of IL-6 in DC2.4 cells towards LPS stimulation was significantly increased. However, after pretreatment with multi-GO, the release of IL-6 in DC2.4 cells treated with LPS was significantly inhibited (Figures 4C, D). Simultaneously, after pretreatment with mono-GO or multi-GO, the release of TNF- $\alpha$ from DC2.4 cells in response to LPS was significantly increased (Figures 4E-H). These results indicated that GO was immunotoxic to DC2.4 cells in vitro and was able to cause DCs dysfunction.

\section{RNA-Seq Analysis}

We also conducted experiments at the transcriptome level and found that many genes in DC2.4 cells were up-regulated and downregulated after mono-GO and multi-GO treatment. Through 

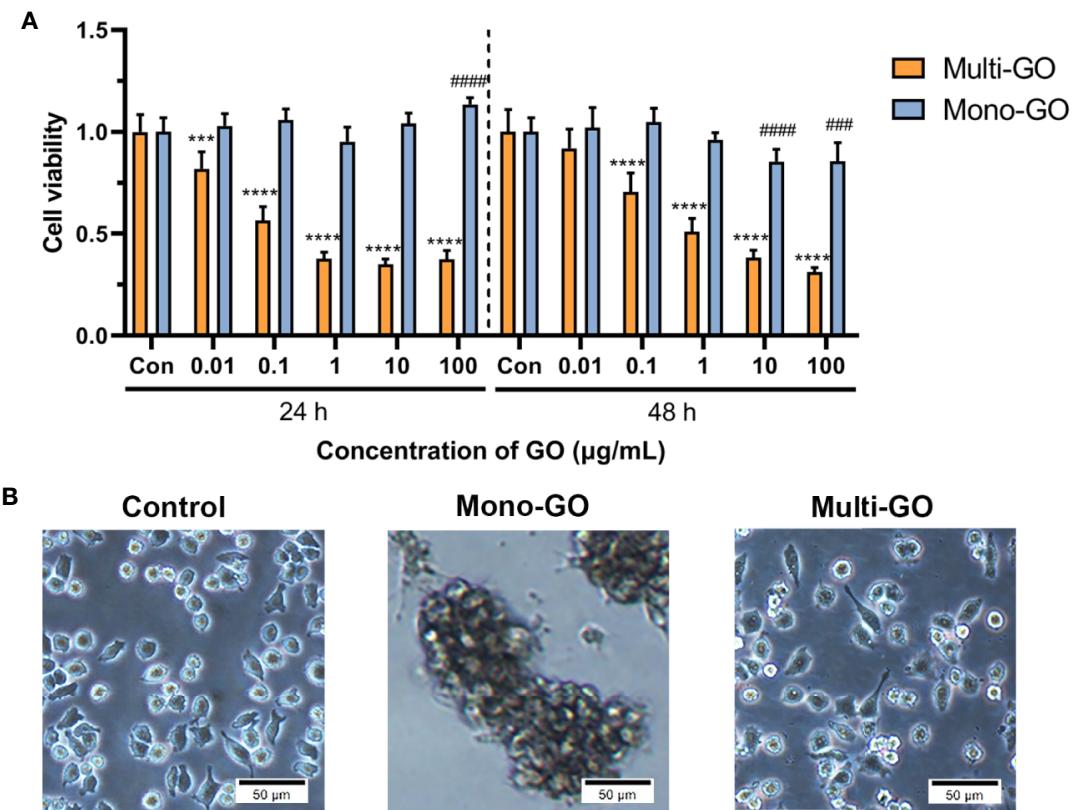

Mono-GO

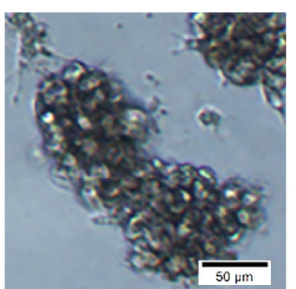

Multi-GO

C

Control
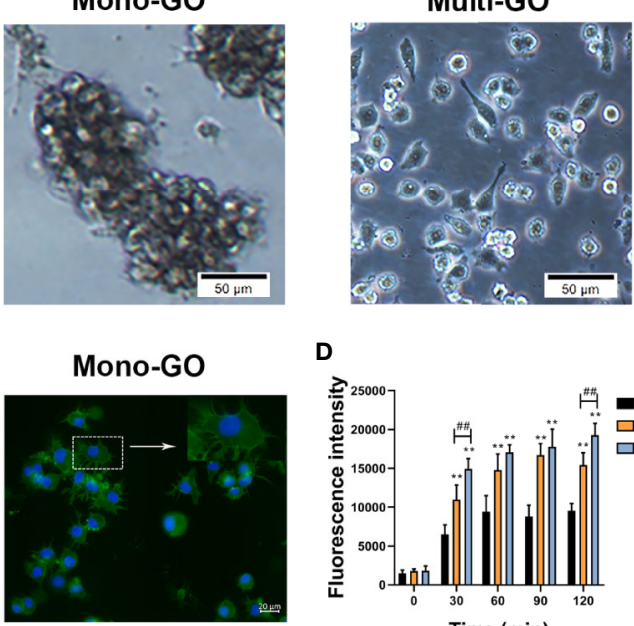

D
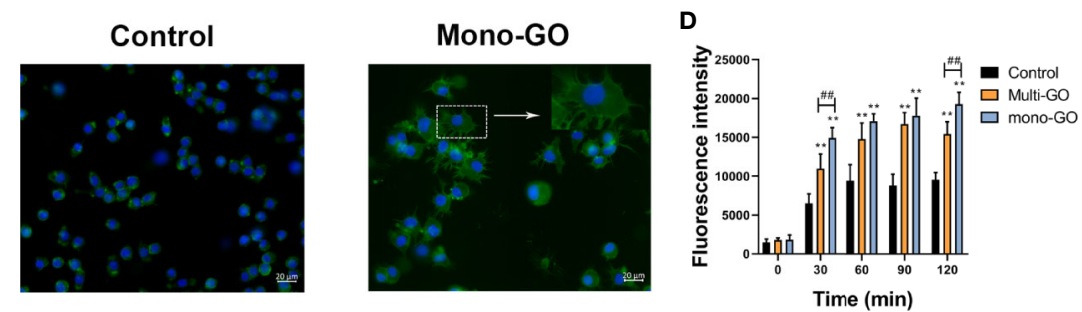

FIGURE 2 | The effect of graphene oxide (GO) on the viability and morphology of DC2.4 cells. (A) The effect of mono-GO and multi-GO on the viability of DC2.4 cells for $24 \mathrm{~h}$ and 48 h. $\mathrm{n}=10$. ${ }^{{ }^{\star \star} \mathrm{P}}<0.001$, ${ }^{\star \star \star \star} \mathrm{P}<0.0001$ multi-GO vs. Control; ${ }^{\# \# \#} \mathrm{P}<0.001$, ${ }^{\# \# \# \# ~} \mathrm{P}<0.0001$ mono-GO vs. Control. (B) Representative brightfield images of DC2.4 cells treated with phosphate-buffered saline (PBS), $10 \mu \mathrm{g} / \mathrm{ml}$ mono-GO, and $10 \mu \mathrm{g} / \mathrm{ml}$ multi-GO. Scale bar $=50 \mu \mathrm{m}$. (C) Representative fluorescent picture of DC2.4 treated with control and mono-GO after phalloidin-FITC staining. Scale bar $=20 \mu \mathrm{m}$. (D) The production of reactive oxygen species (ROS) in DC2.4 cells treated with multi-GO and mono-GO within 120 min. ( ${ }^{\star \star P} P<0.01$ vs. Control; ${ }^{\# \# P}<0.01$ vs. Multi-GO).

analysis, we found that the top 20 genes that significantly changed after mono-GO and multi-GO treatment of DC2.4 cells were Hsp90ab1, Rplp0, Hspa8, Hdgf, Actb, Ldha, Eef1a1, Rpl4, Fth1, Ftl1, Pkm, P2rx7, Eef2, Pabpc1, Gpnmb, Spp1, LOC108169013, Ncl, Eif4g2, and Lcp1. A Principal Component Analysis (PCA) on transcripts was used to assess the quality of the RNA-seq data. In the PCA plot (Figure 5A), three treatments cluster separately from the others. As shown in Figure 5B, the volcano map revealed significant changes in the expression level of 1239 genes between mono-GO group and control group (519 up-regulated genes, 720 down-regulated genes, $\mathrm{P}<0.05, \log _{2}$ fold change $>1$ or $\log _{2}$ fold change $<-1$ ). As shown in Figure 5C, the volcano map also revealed significant changes in the expression level of 261 genes between multi-GO group and control group (91 up-regulated genes, 170 down-regulated genes, $\mathrm{P}<0.05, \log _{2}$ fold change $<-1$ or $>1$ ). Functional annotations against the mouse database revealed series of altered transcriptions involved in BP, CC and MF (Figures 5D, E). These results suggested that mono-GO and multi-GO treatment would lead to obvious changes in gene expression profile. In addition, we conducted a KEGG pathway analysis, and the results are displayed in the form of a bubble chart (Figures 5F, G). It was shown that mono-GO changed many pathways, such as Th1 and Th2 cell differentiation, IL-17 signaling pathway, cytokine-cytokine receptor interaction, ribosome, oxidative phosphorylation and inflammatory bowel disease (IBD), etc. However, the signal pathways changed by multi-GO were different from mono-GO, such as cysteine and methionine metabolism, asthma, synaptics vesicle cycle, and intestinal immune network for IgA production, etc.

In order to analyze the similarities and differences of the effects of mono-GO and multi-GO on immune system process (GO: 0002376), the Venn diagram shown in Figures 6A, B plots the overlapping of differentially expressed genes from immune system processes between different treatment groups. Compared with multi-GO treatment group, the mono-GO treatment group had greater influence on the immune response process of DC2.4 cells. We identified 7 shared downregulated genes and 1 shared upregulated gene between mono-GO vs. control and multi-GO vs. control. The shared 


\section{IL-6}

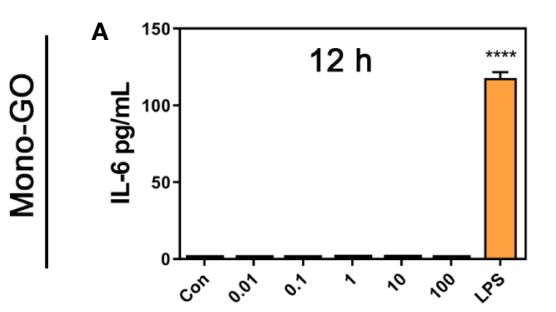

Concentration of mono-GO $(\mu \mathrm{g} / \mathrm{mL})$
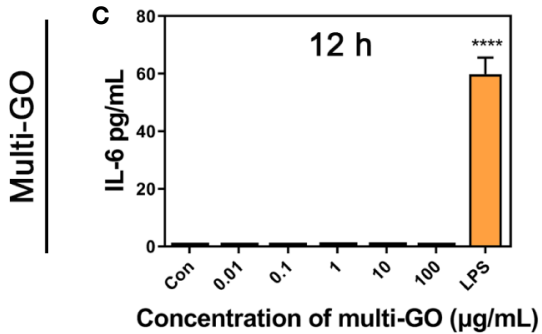

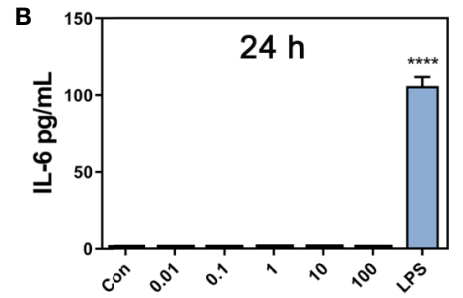

Concentration of mono-GO $(\mu \mathrm{g} / \mathrm{mL})$

D

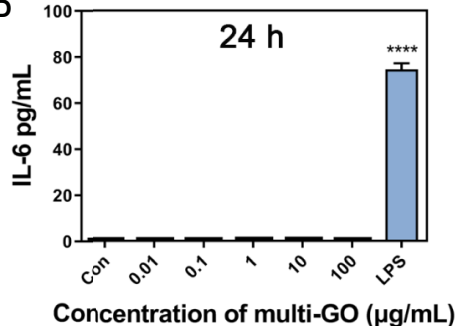

TNF- $\alpha$

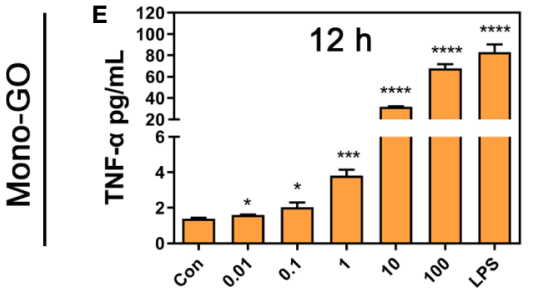

Concentration of mono-GO $(\mu \mathrm{g} / \mathrm{mL})$

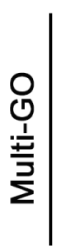

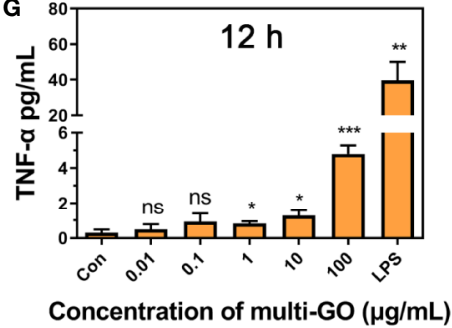

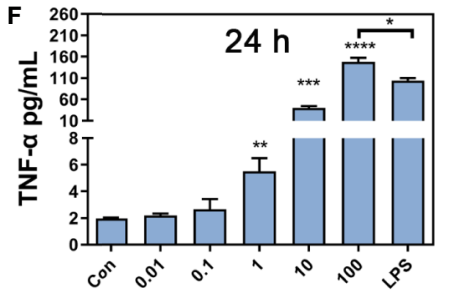

Concentration of mono-GO $(\mu \mathrm{g} / \mathrm{mL})$

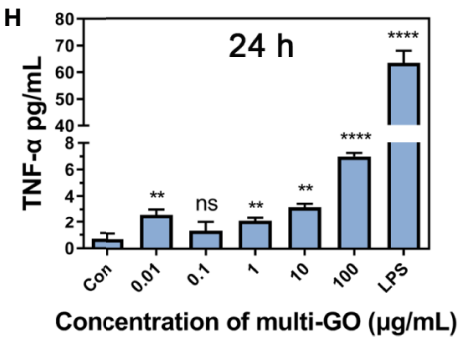

FIGURE 3 | Cytokine secretion of DC2.4 cells exposed to mono-graphene oxide (GO) and multi-GO. (A, B) Cytokine IL-6 secretion from DC2.4 cells after exposure to mono-GO for $12 \mathrm{~h}$ and $24 \mathrm{~h}$. (C, D) Cytokine IL-6 secretion from DC2.4 cells after exposure to multi-GO for $12 \mathrm{~h}$ and $24 \mathrm{~h}$. (E, F) Cytokine TNF- $\alpha$ secretion from DC2.4 cells after exposure to mono-GO for $12 \mathrm{~h}$ and $24 \mathrm{~h}$. (G, $\mathbf{H})$ Cytokine TNF- $\alpha$ secretion from DC2.4 cells after exposure to multi-GO for $12 \mathrm{~h}$ and $24 \mathrm{~h}$. ( $\mathrm{n}=3$; * $\mathrm{P}<0.05,{ }^{\star \star} \mathrm{P}<0.01,{ }^{\star \star \star} \mathrm{P}<0.001,{ }^{\star \star \star \star} \mathrm{P}<0.0001$ vs. Control; ns, no significance).

differentially expressed genes included $\mathrm{H} 2-\mathrm{DMb} 1, \mathrm{Ncbp} 3$, Oas2, Men1, Fas, Cd320, Cd244, and Tinagl1 which play great important role in the immune system process (Figure $6 \mathrm{C}$ ).

\section{DISCUSSION}

In recent years, because of the unique electronic and physicochemical properties of GFNs, they are widely used in various fields, including nanoelectronics and nanobiomedicine (Thangamuthu et al., 2019). However, safety risk is still a great concern for their application and the potential toxicity of human health need to be addressed. Especially, the interaction of GFNs with the immune system remains undefined. DCs are important components of the immune system and play a key role in regulating the body's immune function and maintaining the stability of the body's function. Both the hyperactivity and the underdevelopment of DC's immune function will lead to the imbalance of the body's immune function, leading to the occurrence of diseases. In this work, we aimed to determine the effects of mono-GO and multi-GO on DCs using in vitro cell model. 
IL-6
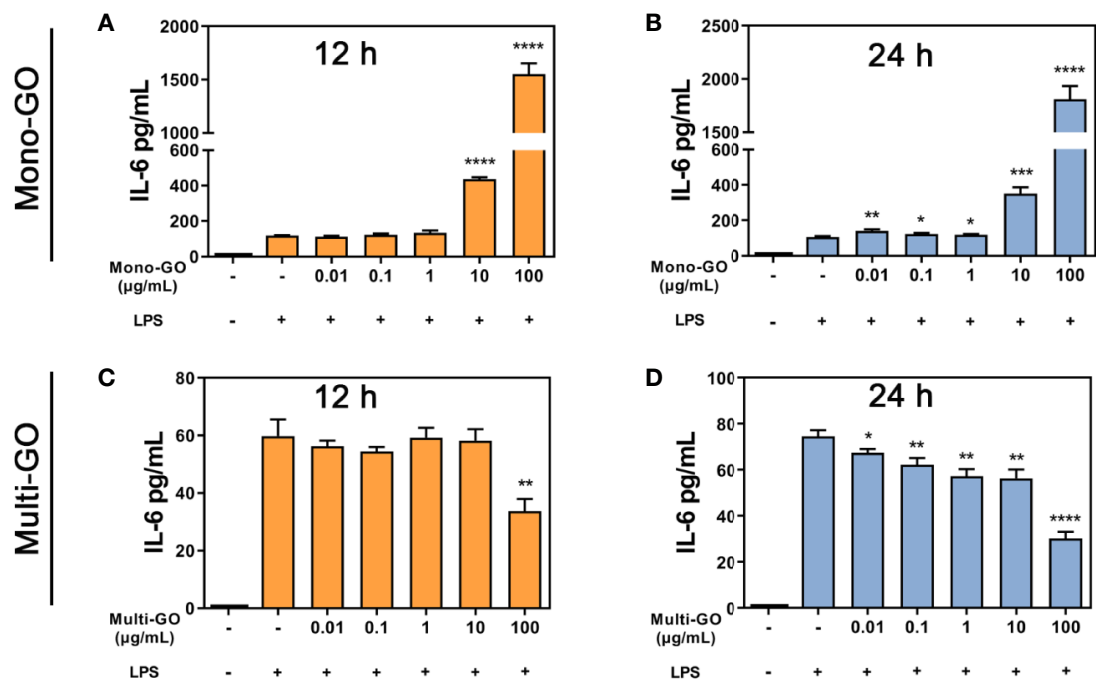

TNF- $\alpha$
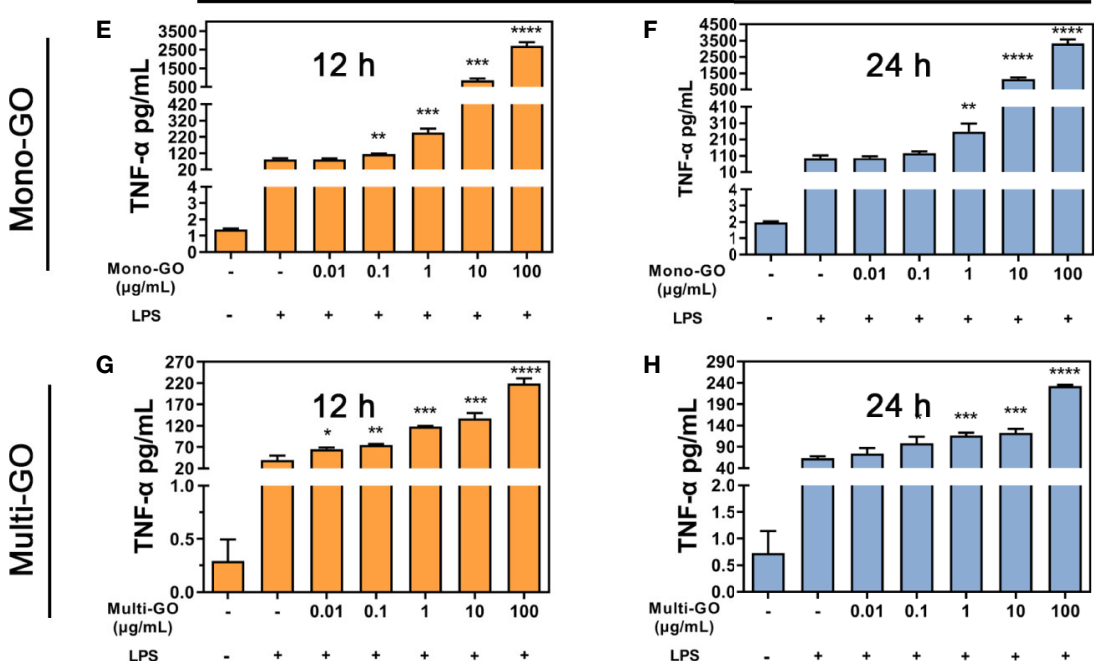

FIGURE 4 | Mono-graphene oxide (GO) or multi-GO promotes or inhibits lipopolysaccharide (LPS)-induced IL-6 and TNF- $\alpha$ cytokine secretion. (A, B, E, F) DC2.4 were treated with mono-GO (10 $\mu \mathrm{g} / \mathrm{ml}, 4 \mathrm{~h})$ before the addition of LPS (1 $\mu \mathrm{g} / \mathrm{ml}, 12 \mathrm{~h}$ or $24 \mathrm{~h} ; \mathrm{n}=3)$. (C, D, G, H) DC2.4 were treated with multi-GO (10 $\mu \mathrm{g} / \mathrm{ml}, 4 \mathrm{~h})$ before the addition of LPS $(1 \mu \mathrm{g} / \mathrm{ml}, 12 \mathrm{~h}$ or $24 \mathrm{~h} ; \mathrm{n}=3)$. ${ }^{*} \mathrm{P}<0.05$; ${ }^{* \star} \mathrm{P}<0.01$; ${ }^{* \star *} \mathrm{P}<0.001$; ${ }^{\star \star \star *} \mathrm{P}<0.0001$ vs. LPS.

According to bioapplication and various toxicity studies of GO (Shao et al., 2017; Hoyle et al., 2018; Pelin et al., 2018; Gurunathan et al., 2019a), we finally selected concentrations of $0.01 \mu \mathrm{g} / \mathrm{ml}, 0.1 \mu \mathrm{g} / \mathrm{ml}, 1 \mu \mathrm{g} / \mathrm{ml}, 10 \mu \mathrm{g} / \mathrm{ml}$, and $100 \mu \mathrm{g} / \mathrm{ml}$ for in vitro toxicity tests. Here, our results showed mono-GO is less toxic to DCs than multi-GO. The cytotoxicity of multi-GO on DC2.4 is concentration dependent after treatment with $24 \mathrm{~h}$ or $48 \mathrm{~h}$. Even at low concentrations $(0.1 \mu \mathrm{g} / \mathrm{ml})$, a high cell proliferation inhibition occurred. Differently, the cell viability of DC2.4 showed no obvious difference after mono-GO treatment for $24 \mathrm{~h}$, but appeared to increase after treatment for $48 \mathrm{~h}$ at the concentration of $100 \mu \mathrm{g} / \mathrm{ml}$. These results indicated that the cytotoxicity of GO on DCs was closely related to the layers and incubation time. Previous reports have proved that many factors are affecting the toxicity of graphene in biological system such as concentration, lateral dimension, layers, surface structure and functional groups (Ou et al., 2016). For example, Peruzynska et al. observed the viability of MCF7 cells exposed to single and four layers GO nanoflakes for $48 \mathrm{~h}$. They found that GO nanoflakes with concentrations lower than $50 \mu \mathrm{g} / \mathrm{ml}$ exhibited no obvious cytotoxicity, but at a concentration of 100 $\mu \mathrm{g} / \mathrm{ml}$ GO the number of living cells significantly reduced, survival rate of $55.5 \%$ for 1 -layer graphene oxide, and $52.9 \%$ for 4-layer graphene oxide, respectively (Peruzynska et al., 2017). 


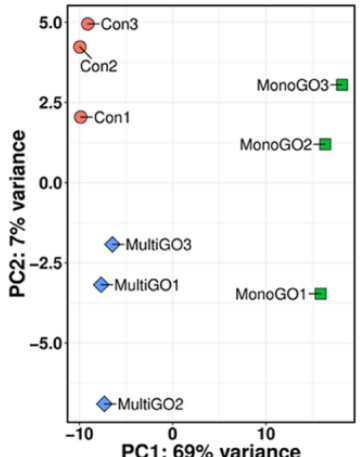

B

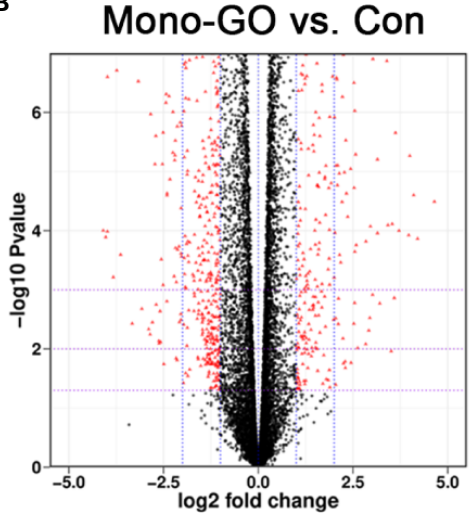

\section{Multi-GO vs. Con}

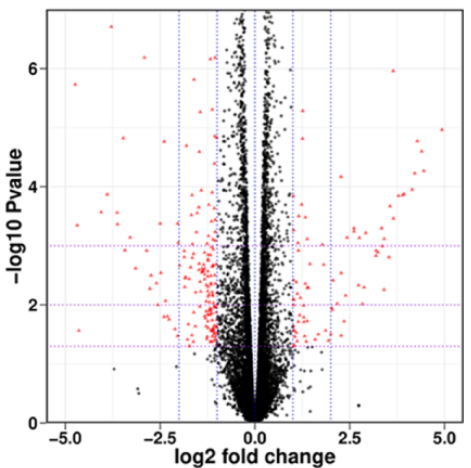

D

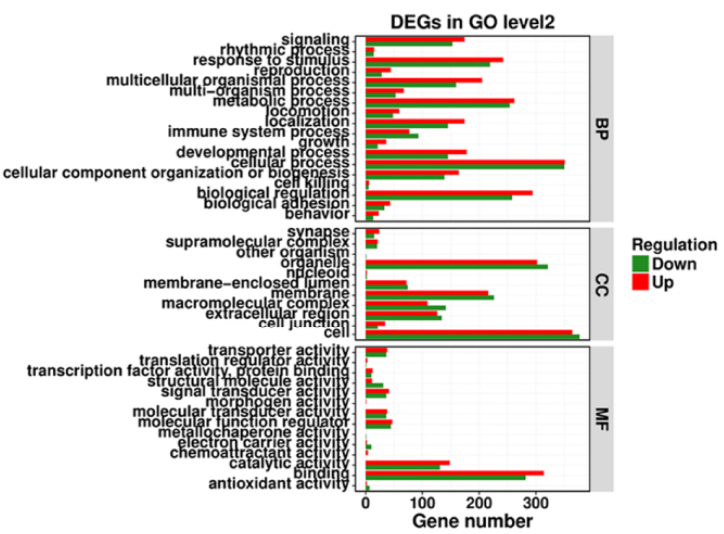

$\mathbf{F}$

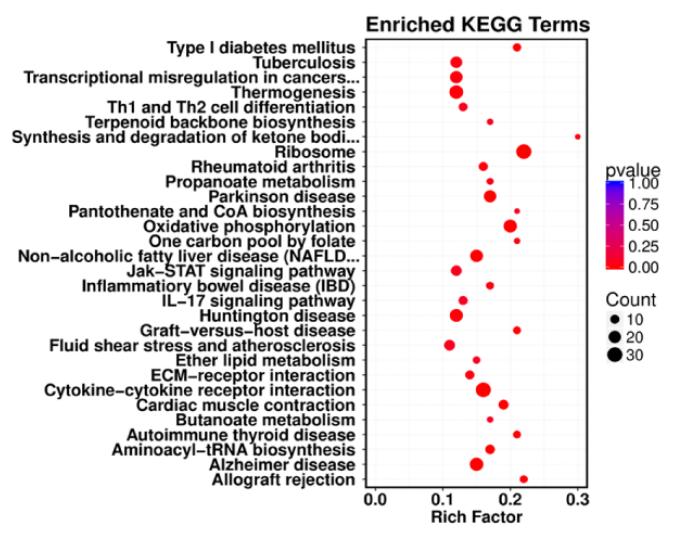

E

Multi-GO vs. Con

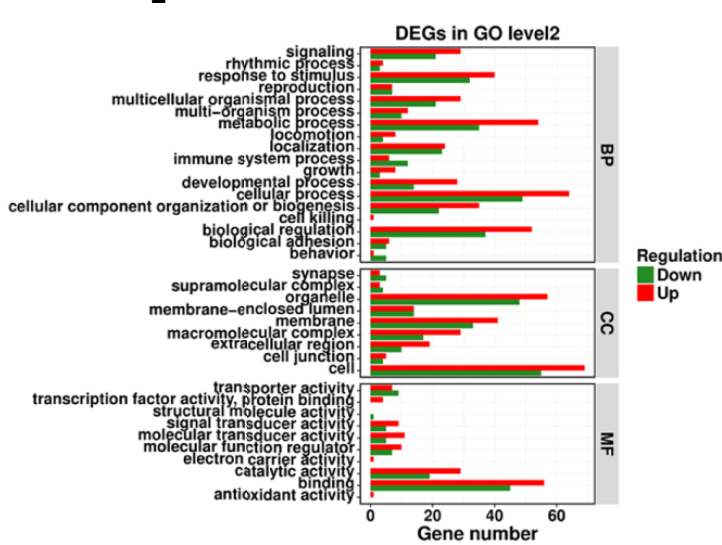

G

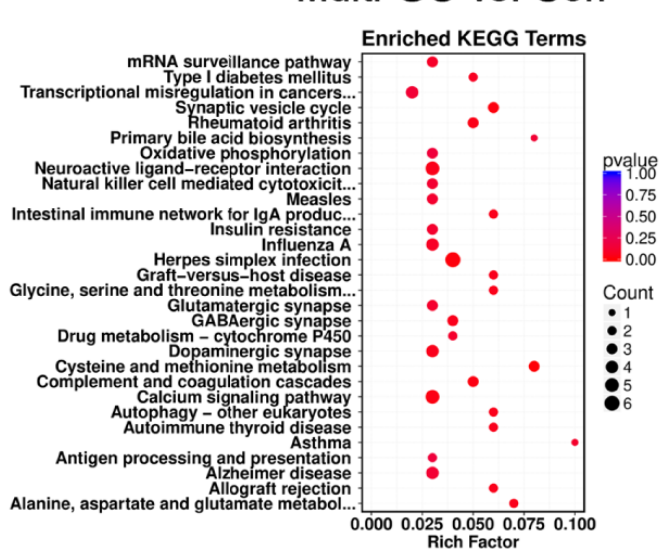

FIGURE 5 | (A) Principal component analysis (PCA) plots including all data, three groups [control, mono-graphene oxide (GO), multi-GO] and their biological replicates; control is in pink, mono-GO is in green and multi-GO is in blue. (B, C) The volcano map shows that all differentially expressed genes compared to the control group after treatment of DC2.4 cells with mono-GO and multi-GO. ( $P<0.05, \log _{2}$ fold change $>1$ or $\log _{2}$ fold change $\left.<-1\right)$. (D, E) DC2.4 cells were analyzed for Gene Ontology (GO) enrichment at level 2 after mono-GO and multi-GO treatments. (F, G) DC2.4 cells were analyzed for KEGG pathway after mono-GO and multi-GO treatments. 


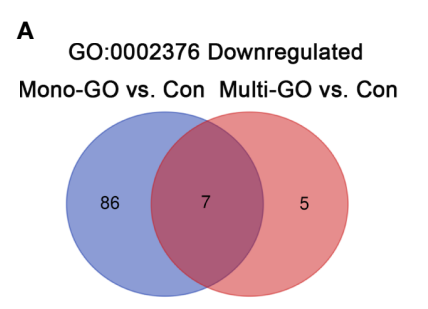

B GO:0002376 Upregulated
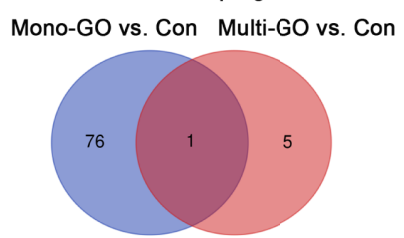

C

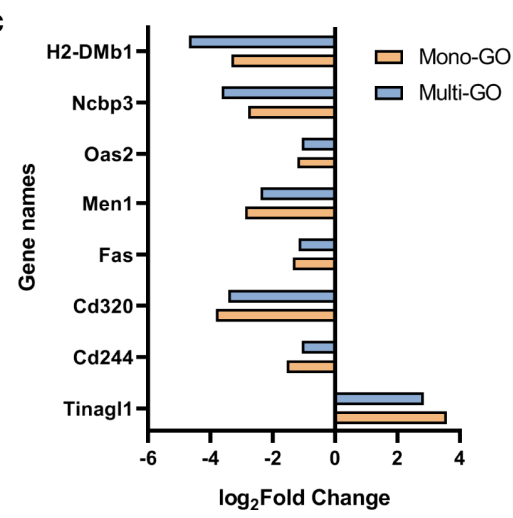

FIGURE 6 | Venn diagram of upregulated and downregulated immune system process (GO: 0002376) after exposure to mono-graphene oxide (GO) and multiGO compared with control. (A) Venn diagram of downregulated immune system process (GO: 0002376). (B) Venn diagram of upregulated immune system process (GO: 0002376) (C) The fold change in differentially expressed genes shared between mono-GO vs. control and multi-GO vs. control.

Jia et al. studied the toxicity of three different sizes (small, medium and large) of graphene (G) and graphene oxide (GO) in vivo and in vitro and they concluded that the toxicity of $\mathrm{G}$ and GO is closely related not only to their inherent chemical properties (oxidation state and side dimensions), but also to the exposure concentration, time, and type of toxicity assessment model used (Jia et al., 2019). Gies et al. systematically evaluated the effects of cell type, exposure time, and sheet size of GO on cytotoxicity, and found that the cytotoxicity of GO is closely related to the cell type (Gies and Zou, 2018). Their data showed that GO had the least toxicity to adherent cells, but the most toxicity to suspension cells. They also investigated the effects of three sizes of GO on the cytotoxicity of different cell types. They found that the cytotoxicity was dependent on GO sheet size for NIH 3T3, U87, and A549 cells, while little effect of sheet size on toxicity for RAW 264.7, NB4, and HL60 cell lines.

Previous research reported that the production of ROS is one of the important mechanisms for the toxicity of nanoparticles (Khatri et al., 2013; Seabra et al., 2014; Tang et al., 2018). Nanoparticles interfere with oxidative balance of cell and cause oxidative stress to generate reactive oxygen species, such as superoxide, hydroxyl radical, peroxide radical, hydrogen peroxide, and singlet oxygen (Yan et al., 2017; Lu et al., 2019). A large amount of ROS can destroy cell structure and cell function, and even cause cell death. Xiong et al. studied the mechanism of the toxicity of GO on zebrafish, and they found that GO-induced liver injury was mainly mediated by ROS and MAPK signals (Xiong et al., 2020). Pelin et al. found that fewlayer-graphene and GO mediated a significant increase in intracellular ROS production primarily through activation of flavin-based oxidase in human HaCaT skin keratinocytes, and caused significant mitochondrial membrane depolarization and mitochondrial damage (Pelin et al., 2018). In our study, the results showed that $10 \mu \mathrm{g} / \mathrm{ml}$ mono-GO and multi-GO caused the production of ROS in DC2.4 cells, and it was a timedependent increase within $2 \mathrm{~h}$. Interestingly, $10 \mu \mathrm{g} / \mathrm{ml}$ monoGO increased intracellular ROS production but did not affect cytotoxicity. The results indicated that ROS generated in a short period of time may not cause cell death. This is similar to previous reports. Chang et al. reported that at lowest concentration of GO was found to induce significant increase in ROS levels in A549 cells without affecting cell viability (Chang et al., 2011). Although the generation of ROS is one of the important mechanisms for cell death caused by nanomaterials, there are still many other cell death-related mechanisms which are responsible for the cytotoxicity induced by nanoparticles such as apoptosis, necrosis, autophagy and necrosis (Ou et al., 2016). In our study, we also found that mono-GO-induced ROS production was more than multi-GO after 30- and 120-min exposure. The reason may be caused by different generation and elimination rates of ROS. Antioxidant enzymes, such as superoxide dismutase or glutathione peroxidase, can reduce and eliminate ROS. The specific mechanism needs further study.

Although mono-GO did not cause cell death, we found an interesting phenomenon in cell morphology. After exposing DC2. 4 cells to $10 \mu \mathrm{g} / \mathrm{ml}$ mono-GO for $24 \mathrm{~h}$, the cell morphology changed, cells began to aggregate and grow, and lost contact inhibition. Staining with ghost pen cyclopeptide revealed that compared with the control group, the cells became larger and the cytoplasm became larger and fuller. $\mathrm{Hu}$ et al. reported that compared with untreated DCs, DC2.4 cells treated with LPS showed a more mature shape, more extensive dendritic formation and wrinkling ( $\mathrm{Hu}$ et al., 2012). Perhaps mono-GO promoted DC2.4 activation into mature DCs. The maturation of DCs will lead to the production and release of cytokines, which will trigger immune defense. Previous research suggested that after human DCs are treated with PLGA nanoparticles (NPs) for $24 \mathrm{~h}$, they produce pro-inflammatory cytokines such as IL-6, IL-8, IL- $\beta$, and TNF- $\alpha$. (Barillet et al., 2019). Conversely, in murine BMDCs, NPs had no effect on cytokines secretion. But when cells were treated with lower concentrations of PLGA NPs, it produced IL-6 and TNF- $\alpha$ In our results, there were no effects on IL- 6 cytokines secretion, but induced TNF- $\alpha$ cytokine secretion after DC2.4 cells incubated with mono-GO and multi-GO for $24 \mathrm{~h}$ and $48 \mathrm{~h}$. And mono-GO induced more secretion of TNF- $\alpha$ cytokines in DC2.4 than multi-GO. TNF- $\alpha$ plays an important role in promoting DCs maturation and immune inflammatory response. Therefore, we speculated that compared with multi-GO, mono-GO could cause a more severe inflammatory response, and then promoted DC2.4 cells to mature. Although study showed that mono-GO and multiGO had different effects on DC2.4 cytotoxicity and cytokine 
production, further parameters such as functional characteristics need to be further evaluated.

Effective antigen presentation and $\mathrm{T}$ cell activation are highly correlated with cytokine release. Previous research showed that LPS binding to TLR4 activates the downstream NFKB signaling pathway, which stimulates DCs to produce TNF- $\alpha$ and IL-6 inflammatory factors and initiates an adaptive immune response (Rhule et al., 2008; O’Neill et al., 2013). So, we investigated whether GO affected the normal cytokines secretory of DC2.4 in response to LPS. Our results clearly suggested that GO disrupted the release of cytokines TNF- $\alpha$ and IL- 6 from DC2.4 cells in response to LPS. We found that mono-GO and multi-GO promoted the release of TNF- $\alpha$ cytokines in LPS-induced DC2. 4 cells. In contrast, mono-GO LPS-induced IL-6 release in DC2.4 cells, but multi-GO inhibits LPS-induced IL-6 release. As previously studied, exposing DCs to GO to achieve responses to TNF- $\alpha$ and IL- 6 may control TH1 response (Barillet et al., 2019), which is consistent with the results of TH1 response induced by delivery of model antigens through PLGA particles (Newman et al., 1998). The decreased level of IL-6 in multi-GO treated cells after LPS stimulation was likely due to the cell viability inhibition caused multi-GO. Overall, our results suggested that GOs changed the inflammatory response of DCs to LPS stimulation. Further investigation is needed to show whether the release of cytokines by GO-induced DCs changes their ability to initiate $\mathrm{T}$-cell dependent immune responses.

In order to further study the immunotoxicity of mono-GO and multi-GO, we performed whole transcriptome sequencing analysis. Recently, high-throughput screening methods have been widely used to assess the toxicity of nanoparticles. Deng et al. employed whole-transcriptome profiling to identify 2116 differentially expressed genes between the zebrafish larvae exposed graphene quantum dots (GQDs) groups and the control group and found that GQDs significantly up-regulated most genes in the acute inflammatory response and detoxification process (Deng et al., 2018). In order to better understand the effects of cadmium telluride (CdTe) QD on central nervous system toxicity, Wu et al. performed a high-throughput sequencing to analyze the changes in the rat hippocampal genome caused by two sizes of CdTe QDs and found that compared to $2.2 \mathrm{~nm}$ CdTe QDs, $3.5 \mathrm{~nm}$ CdTe QDs caused severe inflammatory and immune responses in the hippocampus of rats (Wu T. S. et al., 2018). Our bioinformatics data showed that both mono-GO and multi-GO caused changes in the transcriptome level of DC2.4 cells, and found 1,239 differentially expressed genes (DEGs) were between the monoGO and control and 261 DEGs were between the multi-GO and control. The result suggested that mono-GO exposure might have more severe effects on DC2.4 cells than multi-GO at the transcriptome level. The effect of GO on the transcriptome level of DCs may be related to factors such as the number of layers and lateral size of GO. We also found that 7 shared downregulated genes (H2-DMb1, Ncbp3, Oas2, Men1, Fas, Cd320, and Cd244) and 1 shared upregulated genes (Tinagl1) between mono-GO vs. control and multi-GO vs. control on immune system process (GO: 0002376). All of these genes play great important role in the immune system process. For example, previous study found that activated Fas signaling in DCs induced cytokine secretion and played an important role in adjusting $\mathrm{T}$ cell activation, proliferation, differentiation and inflammation response (Han M. M. et al., 2018; Zu Horste et al., 2018). Based on these results, we inferred that at the transcriptome level, mono-GO produced a stronger immune response than multi-GO on DCs, although they share similar changes for some genes. Gurunathan et al. assessed the immunotoxicity of GO and vanillinfunctionalized GO (V-rGO) on THP-1 cells, a human acute monocytic leukemia cell line and they concluded that V-rGO showed significant effects on immunotoxicity compared to GO because of the sharp edges, chemical composition, charge transfer, different carbon to oxygen ratio, and functional groups present on V-rGO (Gurunathan et al., 2019b). In summary, the immunotoxic response of graphene oxide to cells in this study is closely related to the layers, size, and degree of oxidation of graphene oxide.

In our study, mono-GO and multi-GO both had different degrees of toxicity to DC2.4 cells. Previously, it has been shown that PEGylated nanomaterials are biocompatible and do not cause a severe immune response compared to the original materials. For example, Cicuendez et al. studied the effect of GBNs on macrophages in vitro, and found that the inflammatory response is related to particle size and surface modification (Cicuendez et al., 2020). Smaller-sized nanomaterials induced the pro-inflammatory response of macrophages, while PEGlayted GO did not activate this response. However, the research by Luo et al. contradict these results (Luo et al., 2017). They found that PEGylated GO would not be internalized into cells, but would cause the release of stronger cytokines in peritoneal macrophages compared to GO. Next, they studied the mechanism of GO interaction with cells through atomistic molecular dynamics simulations, and they found GO preferentially adsorb onto and/or partially insert into cell membranes and activated downstream signaling pathways. Further experiments showed that PEGylated GO stimulated the secretion of cytokines by enhancing the integrin $\beta 8$-related signaling pathway. Wu et al. prepared five kinds of PEGlayted GO with differing surface charge and degrees of oxidation and compared their toxicity to ocular surface cells and intraocular cells in vitro (Wu W. et al., 2018). It was found that cytotoxicity was related to the oxidation level of nanomaterials, but the surface charge of nanomaterials had no significant effect. Among them, high oxidative levels of PEGlayted GO resulted in higher cytotoxicity. In summary, PEGlayted GO may reduce the toxicity of cells, but we need to conduct subsequent evaluations of the size, number of layers, and surface charge of PEGlayted GO for better biological applications.

\section{CONCLUSION}

In summary, here we investigated the immunotoxicity of mono-GO and multi-GO on DC2.4 cells. We found that both mono-GO and multi-GO significantly induced ROS production in DC2.4 cells, but mono-GO had less effect on DC2.4 cell viability compared to multiGO. Interestingly, mono-GO enabled DC2.4 cells to aggregate and 
change the cell morphology, while multi-GO had no similar effect. In addition, mono-GO and multi-GO hardly induced the release of the IL-6, while obviously increased the production of TNF- $\alpha$ from DC2.4 cells. Mono-GO and multi-GO promoted TNF- $\alpha$ production in DC2.4 cells induced by LPS. In contrast, mono-GO LPS-induced IL-6 release in DC2.4 cells, but multi-GO inhibits LPSinduced IL-6 release. Gene expression profiling showed that both mono-GO and multi-GO caused changes in the transcriptome level of DC2.4 cells, and mono-GO caused more altered genes than multi-GO. There are some similarities and differences of the effects of mono-GO and multi-GO on immune system process (GO: 0002376). These results indicated that GO had immunotoxicity to DC2.4 cells and was able to cause DCs dysfunction. These results suggest their potential immunotoxicity effects on DCs should be taken into full consideration in their biological applications although GO has many advantages. This is of great significance for the subsequent biological and clinical applications of GO.

\section{DATA AVAILABILITY STATEMENT}

The Illumina reads generated in this study are available at the NCBI Sequence Read Archive browser (http://ncbi.nlm.nih.gov/ sra, accession no. SRR10967877, SRR10967878, SRR10967879, SRR10967880, SRR10967881, SRR10967882, SRR10967883,
SRR10967884 and SRR10967885). We declare that the Raw sequencing data is available through the NCBI Sequence Read Archive under Project ID PRJNA602127 (http://trace.ncbi.nlm. nih.gov/Traces/sra/).

\section{AUTHOR CONTRIBUTIONS}

GL conceived and designed the experiments. ZY, YP, WZ, DX, and DL experimented and collected the data. ZY, TC, and LL performed a statistical analysis of all the data. ZY, TC, and LL analyzed the data and drafted the manuscript. GL and XW made a strict modification of the manuscript. All authors contributed to the article and approved the submitted version.

\section{FUNDING}

The project is supported by the grant from National Natural Science Foundation of China (NSFC) (No. 21677102), the grant from Shenzhen Basic Research Project (No. JCYJ20190808153803952), the grant from Discipline Layout project of Shenzhen Science and Technology innovation committee (JCYJ20170818092553608), and the grant from Guangdong Basic and Applied Basic Research Foundation (NO. 2019A1515110342).

\section{REFERENCES}

Barillet, S., Fattal, E., Mura, S., Tsapis, N., Pallardy, M., Hillaireau, H., et al. (2019). Immunotoxicity of poly (lactic-co-glycolic acid) nanoparticles: influence of surface properties on dendritic cell activation. Nanotoxicology 13 (5), 606-622. doi: $10.1080 / 17435390.2018 .1564078$

Carrow, J. K., Cross, L. M., Reese, R. W., Jaiswal, M. K., Gregory, C. A., Kaunas, R., et al. (2018). Widespread changes in transcriptome profile of human mesenchymal stem cells induced by two-dimensional nanosilicates. Proc. Natl. Acad. Sci. U. S. A. 115 (17), E3905-E3913. doi: 10.1073/pnas.1716164115

Chang, Y., Yang, S. T., Liu, J. H., Dong, E., Wang, Y., Cao, A., et al. (2011). In vitro toxicity evaluation of graphene oxide on A549 cells. Toxicol. Lett. 200 (3), 201210. doi: 10.1016/j.toxlet.2010.11.016

Chen, T., Li, L., Lin, X., Yang, Z., Zou, W., Chen, Y., et al. (2020). In vitro and in vivo immunotoxicity of PEGylated $\mathrm{Cd}$-free $\mathrm{CuInS}_{2} / \mathrm{ZnS}$ quantum dots. Nanotoxicology 14 (3), 372-387. doi: 10.1080/17435390.2019.1708495

Cicuendez, M., Fernandes, M., Ayan-Varela, M., Oliveira, H., Feito, M. J., DiezOrejas, R., et al. (2020). Macrophage inflammatory and metabolic responses to graphene-based nanomaterials differing in size and functionalization. Colloids Surf. B-Biointerfaces 186, 110709. doi: 10.1016/j.colsurfb.2019. 110709

Deng, S., Jia, P. P., Zhang, J. H., Junaid, M., Niu, A. P., Ma, Y. B., et al. (2018). Transcriptomic response and perturbation of toxicity pathways in zebrafish larvae after exposure to graphene quantum dots (GQDs). J. Hazard. Mater. 357, 146-158. doi: 10.1016/j.jhazmat.2018.05.063

Dudek, I., Skoda, M., Jarosz, A., and Szukiewicz, D. (2016). The molecular influence of graphene and graphene oxide on the immune system under in vitro and in vivo conditions. Archivum Immunol. Ther. Exp. 64 (3), 195-215. doi: 10.1007/s00005-015-0369-3

Fu, R. H., Hran, H. J., Chu, C. L., Huang, C. M., Liu, S. P., Wang, Y. C., et al. (2011). Lipopolysaccharide-stimulated activation of murine DC2.4 cells is attenuated by $n$-butylidenephthalide through suppression of the NF-kappaB pathway. Biotechnol. Lett. 33 (5), 903-910. doi: 10.1007/s10529-011-0528-5

Gao, W. (2015). "The Chemistry of Graphene Oxide," in Graphene oxide: reduction recipes, spectroscopy, and applications. Ed. W. Gao (Cham \%@ 978-3-319-15500-5: Springer International Publishing), 61-95.

Gies, V., and Zou, S. (2018). Systematic toxicity investigation of graphene oxide: evaluation of assay selection, cell type, exposure period and flake size. Toxicol. Res. 7 (1), 93-101. doi: 10.1039/c7tx00278e

Gurunathan, S., Arsalan Iqbal, M., Qasim, M., Park, C. H., Yoo, H., Hwang, J. H., et al. (2019a). Evaluation of Graphene Oxide Induced Cellular Toxicity and Transcriptome Analysis in Human Embryonic Kidney Cells. Nanomaterials 9 (7), 969. doi: 10.3390/nano9070969

Gurunathan, S., Kang, M. H., Jeyaraj, M., and Kim, J. H. (2019b). Differential Immunomodulatory Effect of Graphene Oxide and Vanillin-Functionalized Graphene Oxide Nanoparticles in Human Acute Monocytic Leukemia Cell Line (THP-1). Int. J. Mol. Sci. 20 (2), 247. doi: 10.3390/ijms20020247

Han, J., Kim, Y. S., Lim, M. Y., Kim, H. Y., Kong, S., Kang, M., et al. (2018). Dual roles of graphene oxide to attenuate inflammation and elicit timely polarization of macrophage phenotypes for cardiac repair. ACS Nano 12 (2), 1959-1977. doi: 10.1021/acsnano.7b09107

Han, M. M., Hu, R., Ma, J. Y., Zhang, B. H., Chen, C., Li, H. B., et al. (2018). Fas signaling in dendritic cells mediates Th2 polarization in HDM-induced allergic pulmonary inflammation. Front. Immunol. 9, 3045. doi: 10.3389/Fimmu. 2018.03045

Hidalgo, R. S., Lopez-Diaz, D., and Velazquez, M. M. (2015). Graphene oxide thin films: influence of chemical structure and deposition methodology. Langmuir 31 (9), 2697-2705. doi: 10.1021/la5029178

Hoyle, C., Rivers-Auty, J., Lemarchand, E., Vranic, S., Wang, E., Buggio, M., et al. (2018). Small, Thin Graphene Oxide Is Anti-inflammatory Activating Nuclear Factor Erythroid 2-Related Factor 2 via Metabolic Reprogramming. ACS Nano 12 (12), 11949-11962. doi: 10.1021/acsnano.8b03642

Hu, X., Zhang, W., Wang, L., Wan, N., Wang, B., Li, W., et al. (2012). The detailed analysis of the changes of murine dendritic cells (DCs) induced by thymic peptide: pidotimod(PTD). Hum. Vaccin, Immunother. 8 (9), 1250-1258. doi: $10.4161 / \mathrm{hv} .20579$ 
Jia, P. P., Sun, T., Junaid, M., Yang, L., Ma, Y. B., Cui, Z. S., et al. (2019). Nanotoxicity of different sizes of graphene (G) and graphene oxide (GO) in vitro and in vivo. Environ. Pollution 247, 595-606. doi: 10.1016/ j.envpol.2019.01.072

Khatri, M., Bello, D., Gaines, P., Martin, J., Pal, A. K., Gore, R., et al. (2013). Nanoparticles from photocopiers induce oxidative stress and upper respiratory tract inflammation in healthy volunteers. Nanotoxicology 7 (5), 1014-1027. doi: 10.3109/17435390.2012.691998

Li, M. J., Liu, C. M., Xie, Y. B., Cao, H. B., Zhao, H., and Zhang, Y. (2014). The evolution of surface charge on graphene oxide during the reduction and its application in electroanalysis. Carbon 66, 302-311. doi: 10.1016/ j.carbon.2013.09.004

Li, H., Fierens, K., Zhang, Z., Vanparijs, N., Schuijs, M. J., Van Steendam, K., et al. (2016). Spontaneous protein adsorption on graphene oxide nanosheets allowing efficient intracellular vaccine protein delivery. ACS Appl. Mater. Interfaces 8 (2), 1147-1155. doi: 10.1021/acsami.5b08963

Lu, J., Tang, M., and Zhang, T. (2019). Review of toxicological effect of quantum dots on the liver. J. Appl. Toxicol. 39 (1), 72-86. doi: 10.1002/jat.3660

Luo, N., Weber, J. K., Wang, S., Luan, B., Yue, H., Xi, X., et al. (2017). PEGylated graphene oxide elicits strong immunological responses despite surface passivation. Nat. Commun. 8 (1), 14537. doi: 10.1038/ncomms14537

Markovic, M., Kumar, A., Andjelkovic, I., Lath, S., Kirby, J. K., Losic, D., et al. (2018). Ecotoxicology of manufactured graphene oxide nanomaterials and derivation of preliminary guideline values for freshwater environments. Environ. Toxicol. Chem. 37 (5), 1340-1348. doi: 10.1002/etc.4074

Newman, K. D., Samuel, J., and Kwon, G. (1998). Ovalbumin peptide encapsulated in poly(d,l lactic-co-glycolic acid) microspheres is capable of inducing a $\mathrm{T}$ helper type 1 immune response. J. Controlled Release 54 (1), 49-59. doi: 10.1016/S0168-3659(97)00142-9

Novoselov, K. S., Geim, A. K., Morozov, S. V., Jiang, D., Zhang, Y., Dubonos, S. V., et al. (2004). Electric field effect in atomically thin carbon films. Science 306 (5696), 666-669. doi: 10.1126/science.1102896

Nurunnabi, M., Parvez, K., Nafiujjaman, M., Revuri, V., Khan, H. A., Feng, X., et al. (2015). Bioapplication of graphene oxide derivatives: drug/gene delivery, imaging, polymeric modification, toxicology, therapeutics and challenges. RSC Adv. 5 (52), 42141-42161. doi: 10.1039/c5ra04756k

O'Neill, L. A. J., Golenbock, D., and Bowie, A. G. (2013). The history of Toll-like receptors - redefining innate immunity. Nat. Rev. Immunol. 13 (6), 453-460. doi: $10.1038 /$ nri3446

Ou, L., Song, B., Liang, H., Liu, J., Feng, X., Deng, B., et al. (2016). Toxicity of graphene-family nanoparticles: a general review of the origins and mechanisms. Particle Fibre Toxicol. 13 (1), 57. doi: 10.1186/s12989-0160168-y

Pelin, M., Fusco, L., Martin, C., Sosa, S., Frontinan-Rubio, J., GonzalezDominguez, J. M., et al. (2018). Graphene and graphene oxide induce ROS production in human HaCaT skin keratinocytes: the role of xanthine oxidase and NADH dehydrogenase. Nanoscale 10 (25), 11820-11830. doi: 10.1039/ c8nr02933d

Peruzynska, M., Cendrowski, K., Barylak, M., Tkacz, M., Piotrowska, K., Kurzawski, M., et al. (2017). Comparative in vitro study of single and four layer graphene oxide nanoflakes - Cytotoxicity and cellular uptake. Toxicol. Vitro 41, 205-213. doi: 10.1016/j.tiv.2017.03.005

Qian, C., and Cao, X. (2018). Dendritic cells in the regulation of immunity and inflammation. Semin. Immunol. 35, 3-11. doi: 10.1016/j.smim.2017.12.002

Reina, G., Gonzalez-Dominguez, J. M., Criado, A., Vazquez, E., Bianco, A., and Prato, M. (2017). Promises, facts and challenges for graphene in biomedical applications. Chem. Soc. Rev. 46 (15), 4400-4416. doi: 10.1039/c7cs00363c
Rhule, A., Rase, B., Smith, J. R., and Shepherd, D. M. (2008). Toll-like receptor ligand-induced activation of murine DC2.4 cells is attenuated by Panax notoginseng. J. Ethnopharmacol. 116 (1), 179-186. doi: 10.1016/j.jep.2007.11.019

Seabra, A. B., Paula, A. J., de Lima, R., Alves, O. L., and Duran, N. (2014). Nanotoxicity of graphene and graphene oxide. Chem. Res. Toxicol. 27 (2), 159168. doi: $10.1021 / \mathrm{tx} 400385 \mathrm{x}$

Shao, C., Liang, J., He, S., Luan, T., Yu, J., Zhao, H., et al. (2017). pH-Responsive Graphene Oxide-DNA Nanosystem for Live Cell Imaging and Detection. Analytical Chem. 89 (10), 5445-5452. doi: 10.1021/acs.analchem.7b00369

Tang, Z., Zhao, L., Yang, Z., Liu, Z., Gu, J., Bai, B., et al. (2018). Mechanisms of oxidative stress, apoptosis, and autophagy involved in graphene oxide nanomaterial anti-osteosarcoma effect. Int. J. Nanomed. 13, 2907-2919. doi: 10.2147/IJN.S159388

Thangamuthu, M., Hsieh, K. Y., Kumar, P. V., and Chen, G. Y. (2019). Grapheneand graphene oxide-based nanocomposite platforms for electrochemical biosensing applications. Int. J. Mol. Sci. 20 (12), 2975. doi: 10.3390/ ijms 20122975

Wu, T. S., Liang, X., He, K. Y., Wei, T. T., Wang, Y., Zou, L. Y., et al. (2018). Transcriptome analysis of different sizes of 3-mercaptopropionic acidmodified cadmium telluride quantum dot-induced toxic effects reveals immune response in rat hippocampus. J. Appl. Toxicol. 38 (9), 1177-1194. doi: $10.1002 /$ jat.3629

Wu, W., Yan, L., Chen, S., Li, Q., Gu, Z., Xu, H., et al. (2018). Investigating oxidation state-induced toxicity of PEGylated graphene oxide in ocular tissue using gene expression profiles. Nanotoxicology 12 (8), 819-835. doi: 10.1080/ 17435390.2018.1480813

Xiong, G., Deng, Y., Liao, X., Zhang, J., Cheng, B., Cao, Z., et al. (2020). Graphene oxide nanoparticles induce hepatic dysfunction through the regulation of innate immune signaling in zebrafish (Danio rerio). Nanotoxicology 14 (5), 667-682. doi: 10.1080/17435390.2020.1735552

Yan, X., Yang, W., Shao, Z., Yang, S., and Liu, X. (2017). Triggering of apoptosis in osteosarcoma cells by graphene/single-walled carbon nanotube hybrids via the ROS-mediated mitochondrial pathway. J. Biomed. Mater. Res. Part A 105 (2), 443-453. doi: 10.1002/jbm.a.35918

Zhang, L., Peng, J., Hong, M. F., Chen, J. Q., and Qiu, J. D. (2018). A facile graphene oxide-based fluorescent nanosensor for in situ "turn-on" detection of telomerase activity. Analyst 143 (10), 2334-2341. doi: 10.1039/c8an00402a

Zhou, C. C., Liu, S. K., Li, J. L., Guo, K., Yuan, Q., Zhong, A. M., et al. (2018). Collagen functionalized with graphene oxide enhanced biomimetic mineralization and in situ bone defect repair. ACS Appl. Mater. Interfaces 10 (50), 44080-44091. doi: 10.1021/acsami.8b17636

Zu Horste, G. M., Przybylski, D., Schramm, M. A., Wang, C., Schnell, A., Lee, Y., et al. (2018). Fas promotes T helper 17 cell differentiation and inhibits Thelper 1 cell development by binding and sequestering transcription factor STAT1. Immunity 48 (3), 556-55+. doi: 10.1016/j.immuni.2018.03.008

Conflict of Interest: The authors declare that the research was conducted in the absence of any commercial or financial relationships that could be construed as a potential conflict of interest.

Copyright (c) 2020 Yang, Pan, Chen, Li, Zou, Liu, Xue, Wang and Lin. This is an open-access article distributed under the terms of the Creative Commons Attribution License (CC BY). The use, distribution or reproduction in other forums is permitted, provided the original author(s) and the copyright owner(s) are credited and that the original publication in this journal is cited, in accordance with accepted academic practice. No use, distribution or reproduction is permitted which does not comply with these terms. 